\title{
NUMERICAL APPLICATION OF KNOT INVARIANTS AND UNIVERSALITY OF RANDOM KNOTTING
}

\author{
TETSUO DEGUCHI \\ Department of Physics, Ochanomizu University \\ Ohtsuka 2-1-1, Bunkyo-ku, Tokyo 112, Japan \\ E-mail:deguchi@phys.ocha.ac.jp \\ KYOICHI TSURUSAKI \\ Department of Physics, University of Tokyo \\ Hongo 7-3-1, Bunkyo-ku, Tokyo 113, Japan \\ E-mail: turusaki@monet.phys.s.u-tokyo.ac.jp
}

\begin{abstract}
We study universal properties of random knotting by making an extensive use of isotopy invariants of knots. We define knotting probability $\left(P_{K}(N)\right)$ by the probability of an $N$-noded random polygon being topologically equivalent to a given knot $K$. The question is the following: for a given model of random polygon how the knotting probability changes with respect to the number $N$ of polygonal nodes? Through numerical simulation we see that the knotting probability can be expressed by a simple function of $N$. From the result we propose a universal exponent of $P_{K}(N)$, which may be a new numerical invariant of knots.
\end{abstract}

1. Introduction. We shall briefly define random knotting probability in terms of random polygons. Let us consider an $N$-noded polygon given in 3 dimensions. We define a configuration of the polygon by a sequence of the position vectors of the nodes (or the vertices) : $\left\{\vec{r}_{j}=\left(x_{j}, y_{j}, z_{j}\right) ; j=1, \ldots, N\right\}$. Let us now assume that a model of random polygon can produce random configurations of $N$-noded polygon. For example, we may take the Gaussian random polygon, which will be introduced in $\S 2$. Suppose that we have $M$ configurations of $N$-noded polygon of the random model. For a given knot $K$ we define knotting probability $P_{K}(N)$ by the fraction of those configurations that have the same knot type $K$; if $M_{K}$ configurations in the $M$ configurations have a knot $K$, then the knotting probability for $K$ is given by $P_{K}(N)=M_{K} / M$.

Recently knotted ring polymers such as knotted DNA are synthesized in chemical and biological experiments $[4,23]$. The formation of knotted species on random ring closure

1991 Mathematics Subject Classification: Primary 82B41; Secondary 57M25.

The paper is in final form and no version of it will be published elsewhere. 
of DNA was observed and their fractions were measured [21, 22]. In statistical mechanics, the knotting probability is important in the study of the entanglement effect of polymers $[9,13,12]$. The knotting probability $P_{K}(N)$ corresponds to the entropy $S_{K}(N)$ of an $N$-noded ring polymer with knot type $K: S_{K}(N)=-k_{B} \log P_{K}(N)+$ const. $\times N$, where $k_{B}$ is the Boltzmann constant. The asymptotic property of random knotting probability has been studied from the viewpoint of mathematics [18]. It is proved that the knot type of an infinitely large random polygon is given by a nontrivial knot $[11,24,25]$. Thus, the problem of random knotting is studied in the different fields of science.

In this paper we discuss the following two problems: how the knotting probability $P_{K}(N)$ behaves as a function of $N$ and how universal the function is. We study the random knotting probability through numerical experiments. The scheme of our experiments is given in the following. Generating a large number of configurations of $N$-noded random polygon by computers, and calculating certain knot invariants for each of the configurations, we can practically enumerate the number $M_{K}$ of those polygons that have the same knot type $K$ : for a given configuration $C$ we search such a knot $K_{1}$ that has the same set of values of the knot invariants with that of $C$, and we assume that the knot type $K$ of $C$ is given by the knot $K_{1}[7]$.

2. Numerical application of knot invariants. Let us briefly review on previous numerical researches on random knotting. For trivial knot $(K=0)$ the knotting probability $P_{0}(N)$ has been evaluated for several different models of random polygon with different lengths $N$ less than about 2000 [28, 10, 17, 3, 15, 16]. For nontrivial knots, however, the knotting probabilities have been evaluated only for short polygons with $N<200$. The authors of the previous numerical works employed only the special values of the Alexander polynomial $\Delta_{K}(t)$ evaluated at certain values of the variable $t$, in particular, the determinant of knot $\left|\Delta_{K}(-1)\right|$.

It seems that it would be practically difficult to calculate knot invariants such as the Alexander and the Jones polynomials for very large polygons, except for the case of determinant of knot $\left|\Delta_{K}(-1)\right|$. When we calculate knot invariants such as knot polynomials for large polygons, there are two technical difficulties: memory-size and computational-time problems [5]. When we calculate polynomial-valued invariants for polygons with large $N$, the required memory size in computation will grow exponentially with respect to $N$ : if we evaluate a term $t^{N}$ for $N=10^{3}$ by putting 2 to $t$, then we have a very large number $2^{1000}$. Furthermore, if we use a straightforward algorithm computing Jones and HOMFLY polynomials, the computation time will grow exponentially with respect to $N[5,14]$.

It has been shown in Ref. [5] through numerical experiment that the derivatives of the Jones polynomial and those of the quantum link invariants are practically useful for numerical application. The method gives a solution to both the computaion time and memory-size problems. Independently, Przytycka and Przytycki have shown that truncated Jones (or HOMFLY, Kauffman) polynomial can be calculated in polynomial time [20]. Recently, Gauss diagram formulas for some Vassiliev-type invariants are introduced [19]. These independent algorithms give methods for computing Vassiliev-type invariants in polynomial time. The application of these algorithms are also interesting future prob- 
lems. Applying the algorithm of the oriented state sum, however, we have investigated the knotting probability of the Gaussian and the rod-bead random polygons for several nontrivial knots [7, 27, 26, 8]. By utilizing oriented state sum models we can calculate the Vassiliev-type invariants derived from the quasi-classical expansion of the quantum knot invariants in polynomial time without using large memory area in computers [5, 7].

For an illustration we give a list of the values of the Alexander polynomial and some Vassiliev-type invariants for some knots.

\begin{tabular}{|c||c||c|c|c|}
\hline \multicolumn{1}{|c||}{ Knot type } & the determinant & \multicolumn{3}{c|}{ the Vassiliev-type invariants } \\
\hline Knot $K$ & $\left|\Delta_{K}(-1)\right|$ & $v_{2}(K)$ & $v_{3}(K)$ & $v_{4}(K)$ \\
\hline \hline $3_{1}(+)$ & 3 & -12 & 60 & -199 \\
\hline $3_{1}(-)$ & 3 & -12 & -36 & -55 \\
\hline $4_{1}$ & 5 & 12 & -12 & 31 \\
\hline $5_{1}(+)$ & 5 & -36 & 276 & -1365 \\
\hline $5_{1}(-)$ & 5 & -36 & -204 & -645 \\
\hline $5_{2}(+)$ & 7 & -24 & 168 & -758 \\
\hline $5_{2}(-)$ & 7 & -24 & -120 & -326 \\
\hline $3_{1}(+) \sharp 3_{1}(+)$ & 9 & -24 & 120 & -254 \\
\hline $3_{1}(+) \sharp 3_{1}(-)$ & 9 & -24 & 24 & -110 \\
\hline $3_{1}(-) \sharp 3_{1}(-)$ & 9 & -24 & -72 & 34 \\
\hline $3_{1}(+) \sharp 4_{1}$ & 15 & 0 & 48 & -312 \\
\hline $3_{1}(-) \sharp 4_{1}$ & 15 & 0 & -48 & -168 \\
\hline $4_{1} \sharp 4_{1}$ & 25 & 24 & -24 & 208 \\
\hline
\end{tabular}

Table I. The values of the determinant of knot $\left|\Delta_{K}(-1)\right|$ and those of the $j$-th coefficient $v_{j}(K)$ in the quasi-classical expansion of Jones polynomial $(j=2,3$, and 4$)$. Symbols $3_{1}(+)$ and $3_{1}(-)$ denote the mirror images of the trefoil. Symbol $K_{1} \sharp K_{2}$ denotes the product of $K_{1}$ and $K_{2}[5,7]$.

3. Models of random polygon. Let us introduce two different types of models of random polygon, the Gaussian random polygon and the rod-bead random polygon. The former is given by ideal chains with no excluded volume (no thickness of polymer), while the latter consists of real chains with the excluded volume. We can study the selfavoiding effect on the knotting probability through simulations on the rod-bead models with different values of the bead radius [8].

Let us explain the random polygons more explicitly. We define jump vector $\vec{u}_{j}$ by $\vec{r}_{j}-$ $\vec{r}_{j-1}$. Then the position vector $\vec{r}_{j}$ of the $j$ th node is given by the sum of the jump vectors: $\vec{r}_{j}=\vec{u}_{1}+\cdots+\vec{u}_{j}$. A configuration of the polygon is specified by its jump vectors. For the Gaussian random polygon, the probability distribution $P\left(\vec{u}_{1}, \ldots, \vec{u}_{N}\right)$ for a configuration $\vec{u}_{1}, \ldots, \vec{u}_{N}$ is given by

$$
P\left(\vec{u}_{1}, \ldots, \vec{u}_{N}\right)=A \exp \left(-\sum_{j=1}^{N} \vec{u}_{j}^{2}\right) \delta\left(\vec{u}_{1}+\cdots+\vec{u}_{N}\right),
$$

where the constant $A$ is the normalization factor and $\delta(x)$ is the Dirac delta function [10].

Let us consider the rod-bead polygon [3]. It has a bead with radius $r$ on each of the vertices of the polygon. All the jump vectors have a unit length $\left(\left|\vec{u}_{j}\right|=1\right)$, and they 
have a rigid constriant that any pair of the vertices should be separated larger than $2 r$ : $\left|\vec{r}_{j}-\vec{r}_{k}\right|>2 r$ for any $j, k$; if there is a pair of vertices $m$ and $n$ with $\left|\vec{r}_{m}-\vec{r}_{n}\right|<2 r$, then the whole configuration of the polygon is not allowed and discarded. [3] The condition $\left|\vec{r}_{j}-\vec{r}_{k}\right|>2 r$ for any vertices $j$ and $k$ realizes the rigidity of the beads of the polygon. The jump vectors can have any directions with equal probability as far as the rigidity constriant is satisfied. The parameter $r$ expresses the excluded volume or the thickness of the polygon.

4. Numerical results. Let us now consider our numerical results in Ref. [7, 27, 26, 8]. We introduce the following formula [7]

$$
P_{K}(N)=C(K)(N / N(K))^{m(K)} \exp (-N / N(K)),
$$

where $m(K), N(K)$ and $C(K)$ are fitting parameters. We call it scaling formula. Applying the scaling formula to the numerical data we see that it gives good fitting curves to the graphs of $P_{K}(N)$ versus $N$ both for the Gaussian random polygon and the rod-bead random polygon $[7,27,26,8]$. Here we consider $N$-noded polygons with $N$ in the region: $50 \leq N \leq 2000$ or 4000 , and also consider the cases of several nontrivial knots, for example, some prime knots such as $3_{1}, 4_{1}$, and some composite knots such as $3_{1} \sharp 3_{1}, 3_{1} \sharp 4_{1}$ and $3_{1} \sharp 3_{1} \sharp 3_{1}$.

In Fig. 1, the numerical values of the knotting probability $P_{K}(N)$ of the Gaussian random polygon are plotted against the step number $N$ (the number of nodes) for three nontrivial knots $3_{1}, 3_{1} \sharp 3_{1}$, and $3_{1} \sharp 3_{1} \sharp 3_{1}$. In Figs. 2,3 , and 4 , the data of $P_{K}(N)$ versus $N$ of the rod bead models with three different values of the radius $r=0.05,0.10$ and 0.15 are plotted, respectively. They are for those of two nontrivial knots $3_{1}$ and $3_{1} \sharp 3_{1}$.

\section{Gaussian Random Polygon}

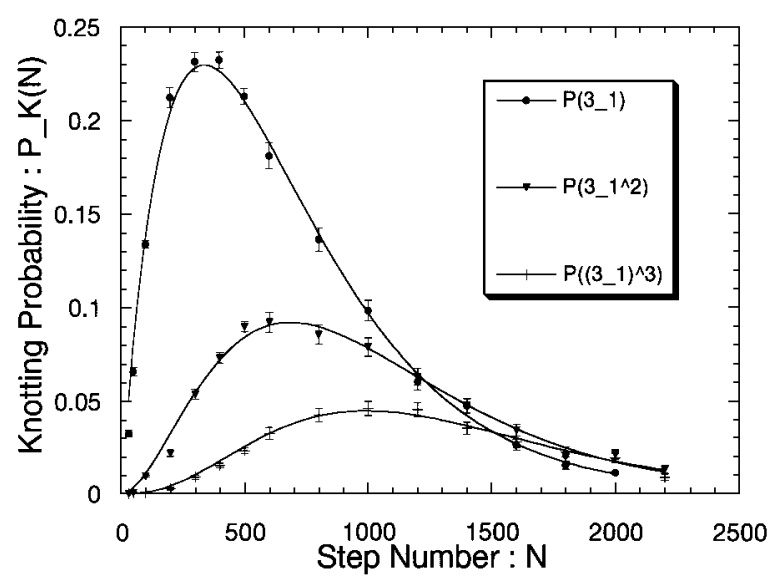

Fig. 1. Random knotting probability of the Gaussian random polygon for $K=3_{1}, 3_{1} \sharp 3_{1}$ and $3_{1} \sharp 3_{1} \sharp 3_{1}$. Large dots denote the numerical values of the knotting probability $P_{K}(N)$ for $K=3_{1}$. The error bars denote the standard deviations of the binomial distribution. The parameters of the fitting curves are given in Table II. 
Rod bead model $r=0.05$

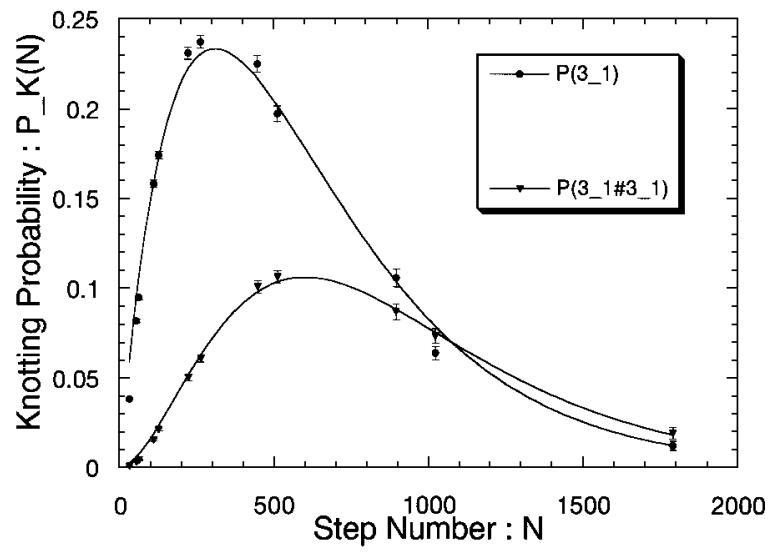

Fig. 2. Random knotting probability of the rod-bead random polygon with the radius $r=0.05$, for $K=3_{1}, 3_{1} \sharp 3_{1}$. Large dots denote the data of the knotting probability $P_{K}(N)$ for $K=3_{1}$. The error bars denote the standard deviations.

Rod-bead model $r=0.10$



Fig. 3. Random knotting probability of the rod-bead random polygon with the radius $r=0.10$, for $K=3_{1}, 3_{1} \sharp 3_{1}$. Large dots denote the data of the knotting probability $P_{K}(N)$ for $K=3_{1}$. The error bars denote the standard deviations.

From Figs. 1, 2, 3 and 4, we find that fitting curves given by the scaling formula are good. Here, the parameters of the fitting curves in Figs. 1, 2, 3, and 4 are given in Table II. We see that the values of $N(K)$ are different for the different values of the bead radius; Fig. 2 shows the data for $r=0.05$ with $N<2000$ which give $N(K) \approx 300$, while Fig. 4 shows the data for $r=0.15$ with $N<4000$ which give $N(K) \approx 900$. Thus, the fitting curves are good for large polygons with $N>100$. However, we should note that the fitting is not very good for small polygons such as those with $N<100$ [8]. This discrepancy 


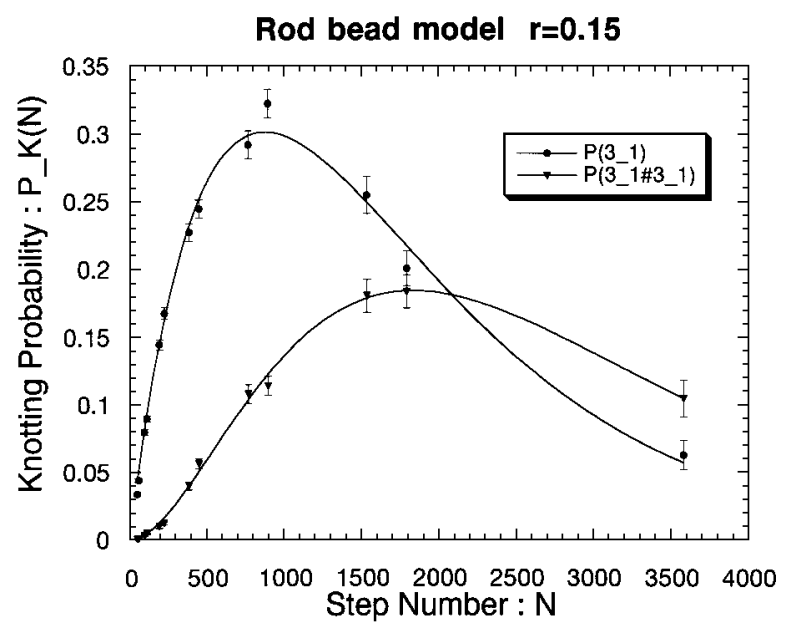

Fig. 4. Random knotting probability of the rod-bead random polygon with the radius $r=0.15$, for $K=3_{1}, 3_{1} \sharp 3_{1}$. Large dots denote the data of the knotting probability $P_{K}(N)$ for $K=3_{1}$. The error bars denote the standard deviations.

may be due to the discreteness of the models of random polygon. As a summary, we may conclude that the random knotting probability for large polygons with $N>100$ can be expressed by the scaling formula, while that for small polygons with $N<100$ can be model-dependent.

\begin{tabular}{|c|c|c||c|c|}
\hline model & knot $K$ & $m(K)$ & $C(K)$ & $N(K)$ \\
\hline \hline Gaussian & 0 & 0.0 & $1.05 \pm 0.03$ & $(3.4 \pm 0.1) \times 10^{2}$ \\
\hline Gaussian & $3_{1}$ & 1.0 & $0.62 \pm 0.02$ & $(3.4 \pm 0.3) \times 10^{2}$ \\
\hline Gaussian & $3_{1} \sharp 3_{1}$ & 2.0 & $0.17 \pm 0.02$ & $(3.4 \pm 0.2) \times 10^{2}$ \\
\hline Gaussian & $3_{1} \sharp 3_{1} \sharp 31$ & 3.0 & $0.033 \pm 0.006$ & $(3.3 \pm 0.2) \times 10^{2}$ \\
\hline$r=0.05$ & 0 & 0.0 & $1.06 \pm 0.03$ & $(3.0 \pm 0.3) \times 10^{2}$ \\
\hline$r=0.05$ & $3_{1}$ & 1.0 & $0.63 \pm 0.02$ & $(3.1 \pm 0.3) \times 10^{2}$ \\
\hline$r=0.05$ & $3_{1} \sharp 3_{1}$ & 2.0 & $0.19 \pm 0.02$ & $(3.0 \pm 0.2) \times 10^{2}$ \\
\hline$r=0.10$ & 0 & 0.0 & $1.04 \pm 0.03$ & $(4.2 \pm 0.1) \times 10^{2}$ \\
\hline$r=0.10$ & $3_{1}$ & 1.0 & $0.70 \pm 0.02$ & $(4.3 \pm 0.4) \times 10^{2}$ \\
\hline$r=0.10$ & $3_{1} \sharp 3_{1}$ & 2.0 & $0.25 \pm 0.03$ & $(4.4 \pm 0.3) \times 10^{2}$ \\
\hline$r=0.15$ & 0 & 0.0 & $1.00 \pm 0.03$ & $(9.0 \pm 0.3) \times 10^{2}$ \\
\hline$r=0.15$ & $3_{1}$ & 1.0 & $0.82 \pm 0.02$ & $(8.8 \pm 0.8) \times 10^{2}$ \\
\hline$r=0.15$ & $3_{1} \sharp 31$ & 2.0 & $0.34 \pm 0.04$ & $(9.2 \pm 0.5) \times 10^{2}$ \\
\hline
\end{tabular}

Table II. The fitting parameters $m(K), N(K)$, and $C(K)$ to the knotting probabilities for the Gaussian model and the rod-bead models with the three bead-radii: $r=0.05,0.10$, and 0.15 [8].

Let us consider the parameter $N(K)$. From Table II we see that in a given model of random polygon, the parameters $N(K)$ for the different knots are given by almost the same value: $N(K) \approx N(0)$ for $K=0,3_{1}$ and $3_{1} \sharp 3_{1}$. 
5. Universality of random knotting. The exponent $m(K)$ of knot $K$ does not change for the Gaussian and the rod-bead models with the three different values of the bead radius: $r=0.05,0.10$ and 0.15 [8]. From Figs. 1-4 we can see it directly from the graphs of the knotting probability $P_{K}(N)$ versus the step number $N$ for the different models of random polygon [8]. The fitting parameters given in Table II are calculated by assuming that the exponent $m(K)$ should be the same for the different models of random polygon and also that $m(0)=0.0,3_{1}=1.0,3_{1} \sharp 3_{1}=2.0$, and $3_{1} \sharp 3_{1} \sharp 3_{1}=3.0$.

Let us discuss how the assumption on the exponent is valid. We consider universal fitting curve for the knotting probability. We define renormalized step number $x$ by $x=$ $N / N(K)$, where $N$ is the step number and $N(K)$ is the fitting parameter given in Table II. We also define renormalized knotting probability $p_{K}(x)$ by $p_{K}(x)=P_{K}(N) / C(K)$, where $P_{K}(N)$ is the knotting probability and $C(K)$ is the fitting parameter given in Table II. If the exponent $m(K)$ is universal (i.e., $m(K)$ has the same value for any model of random polygon) and the knotting probability is given by the scaling formula, then the renormalized knotting probability $p_{K}(x)$ and the renormalized step number $x$ should be related by the universal curve for any model of random polygon:

$$
p_{K}(x)=x^{m(K)} \exp (-x) .
$$

The universal curves of $p_{K}(x)$ versus $x$ for $K=3_{1}$ and $K=3_{1} \sharp 3_{1}$ are shown in Fig. 5 and 6 , respectively. All the numerical data of knotting probabilities for the Gaussian and the rod-bead models with the three radii $r=0.05,0.10$ and 0.15 are shown in Figs. 5 and 6 . We see that the universal curves give good fitting. This suggests that the assumption on the exponent should be valid. The error bars for the exponent $m(K)$ could be less than 0.1 .

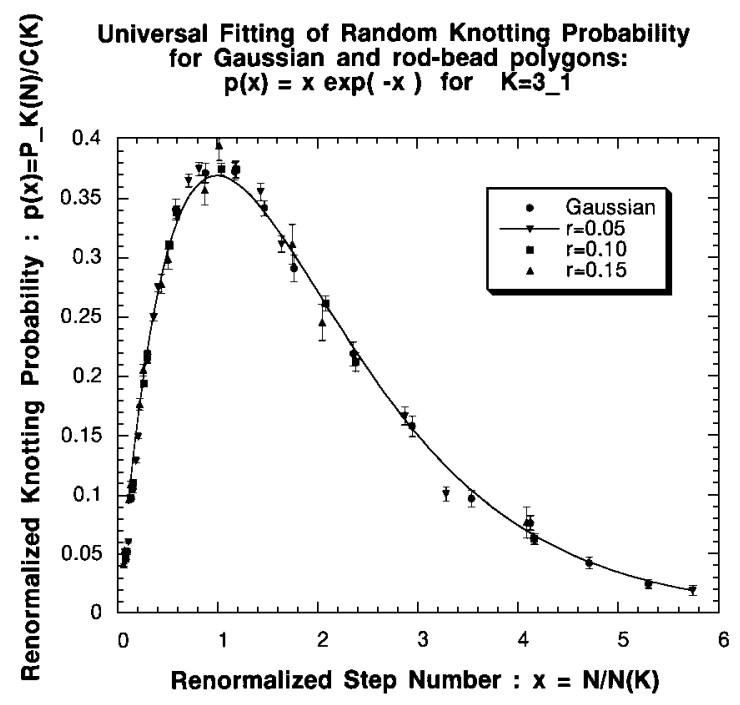

Fig. 5. Universal fitting curve for $K=3_{1}: p_{K}(x)=x \exp (-x)$, and the numerical values of the knotting probability of knot $3_{1}$ for the Gaussian random polygon and the rod-bead random polygons with the three radii $r=0.05,0.10$ and 0.15 . The values for the Gaussian polygon is denoted by large dots. 


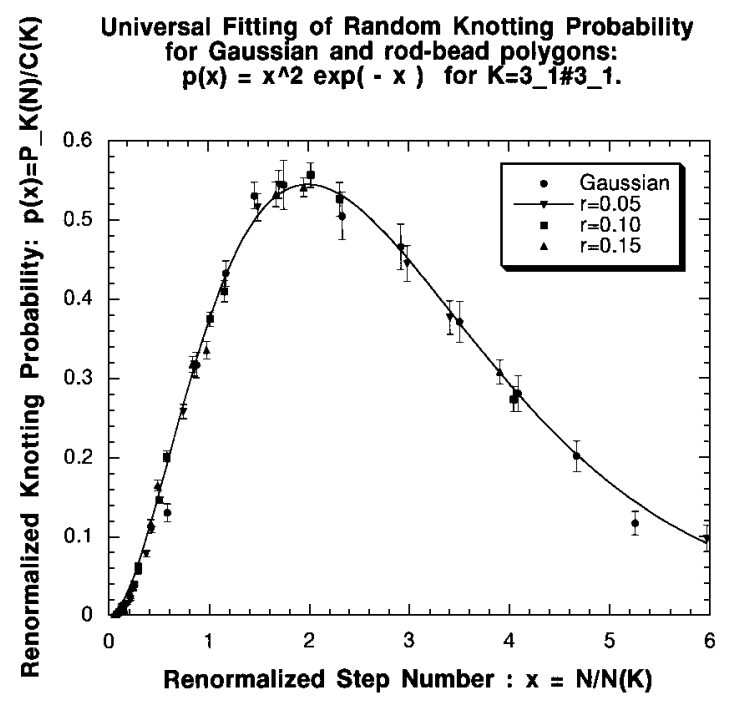

Fig. 6. Universal fitting curve for $K=3_{1} \sharp 3_{1}: p_{K}(x)=x^{2} \exp (-x)$, and the numerical values of the knotting probability of knot $3_{1} \sharp 3_{1}$ for the Gaussian random polygon and the rod-bead random polygons with the three radii $r=0.05,0.10$ and 0.15 . The values for the Gaussian polygon is denoted by large dots.

From the numerical result we propose that the exponent $m(K)$ is universal for each knot type $K$ : for different models of random polygon the knotting probability for knot $K$ is expressed by the scaling formula with the same value of the exponent $m(K)$ and that the exponent $m(K)$ is determined only by the knot type [8].

Let us summarize our numerical result. For a knot $K$ and for a model of random polygon the knotting probability $P_{K}(N)$ is given by

$$
P_{K}(N)=C(K)(N / N(0))^{m(K)} \exp (-N / N(0)),
$$

where the exponent $m(K)$ is universal but $C(K)$ and $N(0)$ are model-dependent. We have a conjecture that the exponent of a composite knot is given by the sum of the exponents of the constituent prime knots: $m\left(K_{1} \sharp K_{2}\right)=m\left(K_{1}\right)+m\left(K_{2}\right)[7,27]$. As far as the numerical results we have obtained, this conjecture together with the above expression of $P_{K}(N)$ is consistent with the data.

Acknowledgments. The authors would like to thank all the organizers of the minisemester on Knot Theory, Stefan Banach International Center, Warsaw, Poland, July 17 -August 18, 1995.

\section{References}

[1] D. Bar-Natan, Topology 34 (1995) 423-472.

[2] J. S. Birman and X. S. Lin, Invent. Math. 111 (1993), 225-270.

[3] Y. D. Chen, J. Chem. Phys. 75 (1981), 2447-2453. 
[4] F. B. Dean, A. Stasiak, T. Koller and N. R. Cozzarelli, J. Biol. Chem. 260 (1985), 4795-4983.

[5] T. Deguchi and K. Tsurusaki, Phys. Lett. A 174 (1993), 29-37.

[6] T. Deguchi and K. Tsurusaki, J. Phys. Soc. Jpn. 62 (1993), 1411-1414.

[7] T. Deguchi and K. Tsurusaki, J. Knot Theory and Its Ramifications 3(1994), 321-353.

[8] T. Deguchi and K. Tsurusaki, A Universality of Random Knotting, preprint 1995.

[9] M. Delbrück, in Mathematical Problems in the Biological Sciences, ed. R.E. Bellman, Proc. Symp. Appl. Math. 14 (1962) 55-63.

[10] J. des Cloizeaux and M. L. Mehta, J. Phys. (Paris) 40 (1979), 665-670.

[11] Y. Diao, N. Pippenger and D. W. Sumners, J. Knot Theory and Its Ramifications 3 (1994), 419-429.

[12] S. F. Edwards, J. Phys. A1 (1968) 15-28.

[13] H. L. Frisch and E. Wasserman, C J. Amer. Chem. Soc. 83 (1961), 3789-3794.

[14] F. Jaeger, D. L. Vertigan and D. J. A. Welsh, Math. Proc. Camb. Phil. Soc. 108 (1990), 35-53.

[15] E. J. Janse van Rensburg and S. G. Whittington, J. Phys. A: Math. Gen. 23 (1990), 3573-3590.

[16] K. Koniaris and M. Muthukumar, Phys. Rev. Lett. 66 (1991), 2211-2214.

[17] J. P. J. Michels and F. W. Wiegel, Phys. Lett. 90A (1982), 381-384.

[18] Random Knotting and Linking, eds. K. C. Millett and D. W. Sumners, World Scientific, Singapore, 1994.

[19] M. Polyak and O. Viro, Intern. Math. Res. Notices, (1994), 445-453.

[20] T. M. Przytycka and J. H. Przytycki, in Graph Structure Theory, eds. N. Robertson and P. Seymour, Contemp. math. AMS 147 (1993), 63-108.

[21] V. V. Rybenkov, N. R. Cozzarelli and A. V. Vologodskii, Proc. Natl. Acad. Sci. USA 90 (1993), 5307-5311.

[22] S. Y. Shaw and J. C. Wang, Science 260 (1993), 533-536.

[23] K. Shishido, N. Komiyama and S. Ikawa, J. Mol, Biol. 195 (1987), 215-218.

[24] D. W. Sumners and S. G. Whittington, J. Phys. A : Math. Gen. 21 (1988), 1689-1694.

[25] N. Pippenger, Discrete Applied Math. 25 (1989), 273-278.

[26] K. Tsurusaki, Thesis: Statistical Study of Random Knotting, University of Tokyo, 1995.

[27] K. Tsurusaki and T. Deguchi, J. Phys. Soc. Jpn. 64 (1995), 1506-1518.

[28] A. V. Vologodskii, A. V. Lukashin, M. D. Frank-Kamenetskii and V. V. Anshelevich, Sov. Phys. JETP 39 (1974), 1059-1063. 